\title{
Design and Real-Time Control of a Robotic System for Fracture Manipulation
}

\author{
G. Dagnino $P h D$, I. Georgilas $P h D$, P. Tarassoli $M D$, R. Atkins $M D$, and S. Dogramadzi $P h D$
}

\begin{abstract}
This paper presents the design, development and control of a new robotic system for fracture manipulation. The objective is to improve the precision, ergonomics and safety of the traditional surgical procedure to treat joint fractures. The achievements toward this direction are here reported and include the design, the real-time control architecture and the evaluation of a new robotic manipulator system. The robotic manipulator is a 6-DOF parallel robot with the struts developed as linear actuators. The control architecture is also described here. The high-level controller implements a hosttarget structure composed by a host computer (PC), a real-time controller, and an FPGA. A graphical user interface was designed allowing the surgeon to comfortably automate and monitor the robotic system. The real-time controller guarantees the determinism of the control algorithms adding an extra level of safety for the robotic automation. The system's positioning accuracy and repeatability have been demonstrated showing a maximum positioning RMSE of $1.18 \pm 1.14 \mathrm{~mm}$ (translations) and $1.85 \pm 1.54^{\circ}$ (rotations).
\end{abstract}

\section{INTRODUCTION}

For optimum healing of bone fractures in the human body, to ensure that the bone and surrounding joints are able to function correctly again, the fragments of the broken bone must be subjected to an anatomical reduction, which involves positioning and aligning the fragments of the broken bone to reconstruct the fractured bone as precisely as possible, so that the bone recovers to a form as close as possible to its original form as it heals [1]. This anatomical reduction may be performed by open surgery, in which large incisions are made in flesh around the broken bone and the bone fragments are manipulated by a surgeon to reposition and realign them as precisely as possible. Whilst this technique can be effective, the damage to the soft tissues and the extensive exposure required by the open surgery procedure typically slows down wound healing and produces unsightly scars, as well as giving rise to an increased risk of infection. A prolonged period of postoperative rehabilitation is required, which requires the patient to endure an extended stay in hospital [2]. In order to mitigate the disadvantages of open surgery techniques, minimally invasive percutaneous procedures have been developed. These techniques involve sequentially fixating and manipulating each bone fragment manually using manipulation pins inserted in the fragments, without

G. Dagnino is with the Bristol Robotics Laboratory, University of the West of England, Coldharbour Lane, Frenchay, BS16 1QY, Bristol, United Kingdom (corresponding author, phone: +44-0117-32-86325; email: giulio.dagnino@uwe.ac.uk).

I. Georgilas, and S. Dogramadzi are with the Bristol Robotics Laboratory, University of the West of England, Coldharbour Lane, Frenchay, BS16 1QY, Bristol, United Kingdom (e-mail: ioannis.georgilas@uwe.ac.uk, sanja.dogramadzi@uwe.ac.uk).

P. Tarassoli and R. Atkins are with the University Hospitals Bristol, UK (email: tarassoli@mail2web.com, R.Atkins@bristol.ac.uk). making large incisions in the patient' s flesh. Such techniques are associated with a faster recovery and a lower risk of infection compared to open surgery techniques [3]. However, minimally invasive techniques have repeatedly shown incomplete reductions due to suboptimal bone/fragment alignment for complex fracture patterns [2] and it remains still unclear in the clinical community as to whether the lower standard of manually performed fracture reduction obtained percutaneously will lead in the long term to early degenerative changes. Furthermore, additional challenges are created by the poor ergonomics of the current setup (Fig.1): minimally invasive procedures require multiple plain radio graphic images of the patient to be taken during the surgical procedure to ensure that the bone fragments are being correctly positioned and orientated during the procedure. The surgeon performs the operation looking at the monitor of the fluoroscopic system to check the accuracy of the bone manipulation, while reducing the fracture. Also, the $2 \mathrm{D}$ nature of this technology doesn't provide enough information to the surgeon regarding the fracture alignment and rotation, which is actually a three-dimensional problem. This scenario constitutes a tiring work setup, which can negatively influence the precision and safety of the surgical procedures [4]. These requirements, together with the issues identified above, lead us to believe that robotic assistance can have a positive impact in this field, allowing more accurate fragment repositioning without open surgery, and obviating problems related to the current percutaneous surgical techniques.

Robot-assisted fracture surgery (RAFS) is potentially able to combine the required high positioning accuracy and repeatability with the minimally invasive approach, hugely reducing in-patient stays and making recovery swifter and more complete. Robot assistance in the operating theatre is currently helping surgeons with new procedures and achieving unprecedented levels of performance [5]. Major technological goals are to improve the surgical outcome,

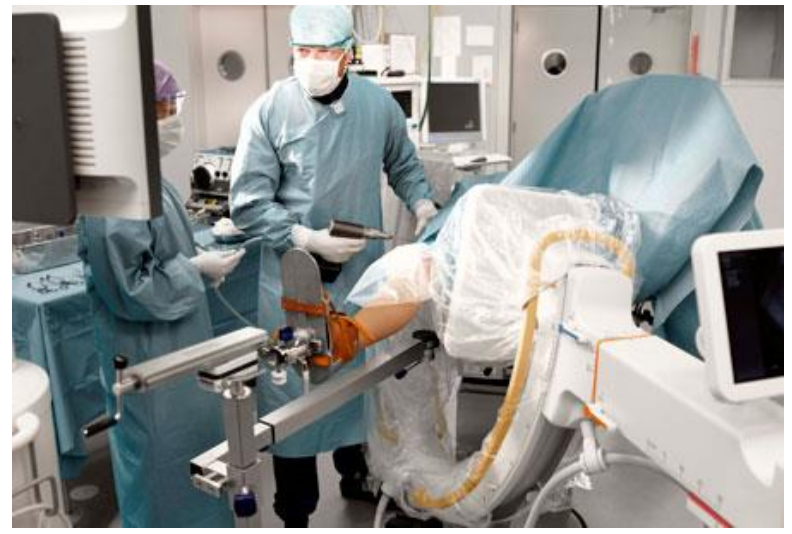

Figure 1. Current setup for minimally invasive fracture surgery. The surgeon operates while checking the reduction on the screen of the fluoroscopic system. 


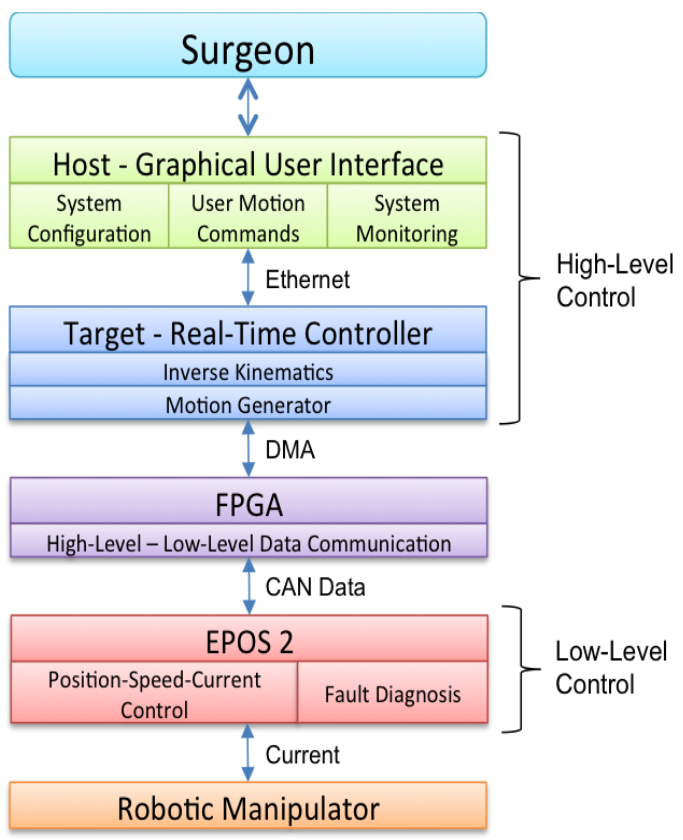

Figure 2. Architecture of the robotic system for fracture manipulation

reduce operating time, and improve patient safety. Robotassisted fracture reduction has been attempted for long bone reduction, specifically femur shaft fractures. Westphal et al. [6] proposed a robotic system for the reduction of femur shaft fractures based on a telemanipulated serial industrial robot. Wang et al. work [7] focused again on femoral shaft fracture reduction using a parallel manipulator robot mounted on a traction table to achieve a better alignment of the fractured femur. Finally, Tang et al. [8] reported on the design and test of a hexapod computer-assisted system for the reduction of diaphyseal femur fractures. However, compared with comminuted fractures, i.e. involving joints, femur shaft fractures have smaller number of larger fragments that require a lower level of manipulation accuracy to be reduced.

In this paper, we present a novel robotic system designed to improve the manipulation accuracy and repeatability of bone fragments during minimally invasive robot-assisted joint fracture surgery. The paper starts with the description of the system setup and specifications (Section II). The robotic manipulator design is presented in Section III followed by the description of the implemented control architecture (Section IV). Results from validation experiments are presented in Section V. Finally, the results are discussed.

\section{SYSTEM CONFIGURATION AND APPLICATION REQUIREMENTS}

The proposed system allows the surgeon to manipulate a bone fragment rigidly attached to the robotic manipulator with high level of accuracy and repeatability. The connection between the robotic manipulator and the fragment is obtained through a metallic orthopedic pin screwed into the fragment by the surgeon and then attached to the manipulator's endeffector. An overview of the developed robotic system is presented in Fig.2. It schematically shows the system structure, with the surgeon in control of the robotic device and planning the surgical procedure from a workstation. The high-level controller processes the surgeon's commands and sends the motion commands to the low-level controller that executes the movement of the robotic manipulator. Specifications for designing this novel surgical system were established through discussions with orthopedic surgeons and analysis of fracture cases [9] [10]. A summary of the parameters that influenced the system design are reported in Table 1.

TABLE I. JoINT FRAGMENT MANIPULATION REQUIREMENTS

\begin{tabular}{ll}
\hline \hline Parameter & Value \\
\hline Translational Accuracy & $<1 \mathrm{~mm}$ \\
Rotational Accuracy & $<5^{\circ}$ \\
Translational Workspace $x, y, z$ & $\pm 10 \mathrm{~mm}$ \\
Rotational Workspace $\vartheta_{x}, \vartheta_{y}, \vartheta_{z}$ & $\pm 15^{\circ}$ \\
Forces/Torques for Manipulating Fragments & $\sim 20 \mathrm{~N}$ (force) \\
\hline \hline
\end{tabular}

\section{ROBOTIC MANIPULATOR DESIGN}

The proposed robotic manipulator is an automated computer-controlled parallel-robot with 6 degrees-of-freedom (DOF), i.e. 3 translations and 3 rotations along/around $x, y, z$ axes (Fig.3a). The use of a parallel-robot is a preferred choice for orthopedic applications [7] [8], where high load carrying capacity and precise positioning accuracy-repeatability are of paramount importance. High precision commercial parallelrobots (such as the H-811 6-axis miniature hexapod by PI $\mathrm{GmbH}$ ) are expensive especially when purpose built to meet the required specifications. Therefore, the parallel-robot here described has been designed and manufactured in-house $a d$ hoc with the following characteristics. The struts have been developed as linear actuators based on a layered design consisting of laser-cut acetal sheets varying in thickness from $1-5 \mathrm{~mm}$. The linear guide elements (ball screws, linear shafts) are made of ground hardened steel allowing for high precision tolerances. The actuation element is a brushed DC motor with integrated gearbox and rotational encoder (RE10MR-GP10K by Maxon Motor) providing high-torque, precise positioning $(0.485 \mu \mathrm{m}$ resolution) while allowing for a simple assembly solution. The 6 linear actuators produce a resulting load capacity of $360 \mathrm{~N}$ (force) and $12 \mathrm{Nm}$ (torque), making the robot suitable also for applications that require a higher load capacity. Table 2 summarizes the design parameters of the parallel-robot, while a picture of the complete setup is shown in Fig.3.

TABLE II. DESIGN PARAMETERS OF THE ROBOTIC MANIPULATOR

\begin{tabular}{ll}
\hline \hline Name & Value \\
\hline Translational Workspace $x-y-z$ & $\pm 10.25 \mathrm{~mm}(x-y)$ \\
Rotational Workspace $\vartheta_{x}-\vartheta_{y}-\vartheta_{z}$ & $\pm 15 \mathrm{~mm}(z)$ \\
Load Capacity & $\pm 17^{\circ}\left(\vartheta_{x}-\vartheta_{y}-\vartheta_{z}\right)$ \\
Moving Platform Diameter & $360 \mathrm{~N}$ (force) \\
Base Platform & $12 \mathrm{Nm}$ (torque) \\
Fully Retracted Strut Length & $118 \mathrm{~mm}$ \\
Fully Extended Strut Length & $135 \mathrm{~mm}$ \\
\hline \hline
\end{tabular}




\section{CONTROL ARCHItecture}

The controller developed for the new robotic manipulator (Fig.3a) employs a host-target structure composed by a host computer, a real-time controller, and a field programmable gate array (FPGA) (see Fig.2). The host-computer creates the link between surgeon and the robot attached to the bone fragment. It runs on a Windows 7 machine with LabView (National Instruments, Austin, TX, USA) to program, operate, and display the graphical user interface (GUI) shown in Fig.3c. Through the GUI the surgeon is able to configure, control, and monitor the system. These features are implemented in three different parallel loops running at $20 \mathrm{~Hz}$. The surgeon can remotely control the robot in automatic mode to drive the robot to a desired pose. In this automatic mode, the surgeon inserts the motion commands into the GUI, i.e. the desired pose for the robot end-effector defined by three translations and three rotations along and around the motion axis, as shown in Fig.3c. The desired pose for the robot is initially calculated based on pre-operative images of the fracture: the surgeon is able to define the fragment translations and rotations required to reduce the fracture by analyzing pre-operative CT images of the fracture. This defines the surgical plan. Then, fluoroscopic imaging is used to check the actual pose of the fragment and the reduction accuracy. Therefore, the surgical plan can be adapted step-by-step intra-operatively to achieve a better reduction. After confirming the surgical plan, the surgeon initiates the automatic motion by pressing a "move" button. The motion command data are sent to the real-time controller (NI-compactRIO 9068, National Instruments Austin, TX, USA) via ethernet. The inverse kinematics and the motion generator algorithms run on the real-time controller (target) at $100 \mathrm{~Hz}$. The inverse kinematics calculates the motion commands for the six robot struts based on the desired robot pose defined by the surgeon, while the motion generator algorithm generate the trajectory for the robot to reach the

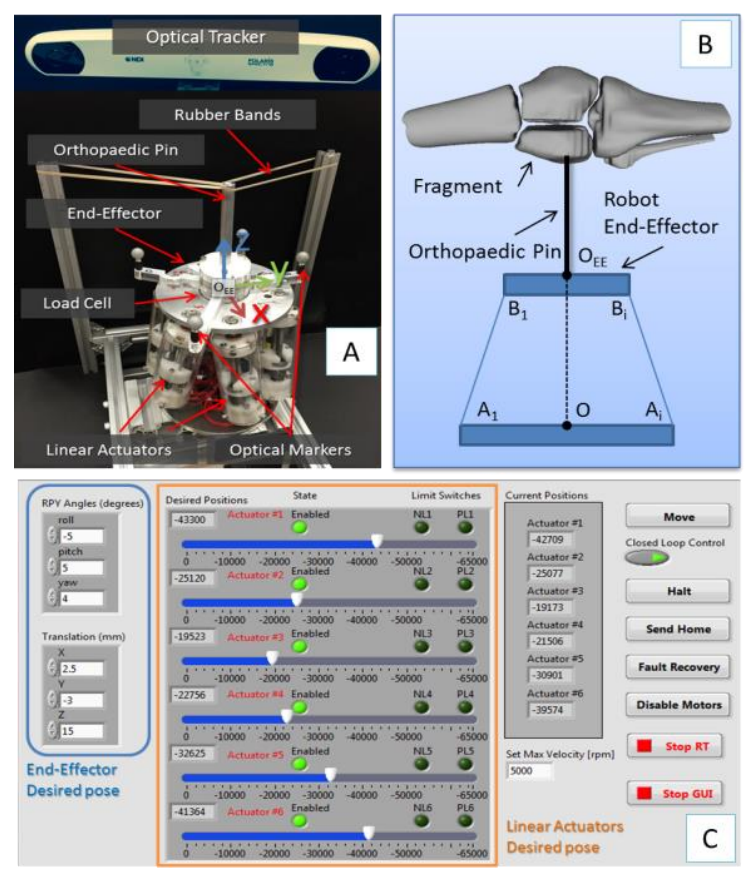

Figure 3. The robotic manipulator and the experimental setup (A), its kinematic model (B), and the control GUI (C). desired pose. The inverse kinematics is derived using the loop closure approach for each strut (Fig.3b). This is a vector method that gives relationships between the key points of the robot structure, i.e. $O$ the center of the base platform, $O_{E E}$ the center of the moving plate and the end-effector, $A_{i}$ and $B_{i}$ are the points where each strut $i$ is attached to the base and the moving platform respectively. Their relation is given in (1) $(i=1 \ldots 6)$.

$$
\overrightarrow{O O_{E E}}=\overrightarrow{O A_{l}}+\overrightarrow{A_{l} B_{l}}+\overrightarrow{B_{l} O_{E E}}
$$

The $\overrightarrow{O O_{E E}}$ vector is the desired position of the endeffector in the reference frame of the base platform, vectors $\overrightarrow{O A_{\imath}}$ and $\overrightarrow{B_{l} O_{E E}}$ are known vectors based on the robot's geometry, usually the diameter of the inscribed circle for the plate and the internal angles of the hexagon. Finally, vector $\overrightarrow{A_{l} B_{l}}$ is the vector of each strut. By representing each point in its three-dimensional vector form, i.e. $\left(x_{n}, y_{n}, z_{n}\right)$ we can calculate the magnitude of this vector using (2) $(i=1 \ldots 6)$.

$$
\left\|\overrightarrow{A_{l} B_{l}}\right\|=\sqrt{\left(x_{B_{i}}-x_{A_{i}}\right)^{2}+\left(y_{B_{i}}-y_{A_{i}}\right)^{2}+\left(z_{B_{i}}-z_{A_{i}}\right)^{2}}
$$

The length values from (2) are the commands delivered to the motion generator that synchronizes the six actuators using a velocity feed-forward control scheme. This is performed by adjusting the axis velocities based on the leading manipulator axis, i.e. the axis performing the slowest motion for a given point-to-point motion step. The velocity profile for each axis is calculated using the following equation $(i=1 \ldots 6)$ :

$$
\dot{\vartheta}_{i}=\frac{\ddot{\vartheta}_{M A X} \cdot t_{e}}{2}-\sqrt{\left(\frac{\ddot{\vartheta}_{M A X}^{2} \cdot t_{e}^{2}}{4}\right)-\left(s_{i, e} \cdot \ddot{\vartheta}_{M A X}^{2}\right)}
$$

where:

$$
t_{e}=\frac{s_{e}}{\dot{\vartheta}_{M A X}}+\frac{\dot{\vartheta}_{M A X}}{\ddot{\vartheta}_{M A X}}
$$

$t_{e}$ is the end time of the trapezoidal velocity profile of the leading robot axis; $s_{e}$ is the maximum axis displacement. This synchronization algorithm is essential to simultaneous control of all robot actuators and to ensure that all actuators start and stop at the same time.

Finally, the synchronized motion data are sent to the FPGA that runs a library dedicated to the communication between the high-level control system and the low-level motor controllers. It generates motion CAN data and sends them to the six low-level controllers (one per actuator), which control the linear actuators to reach the desired pose in the task space. The low-level controllers (EPOS 2 24/3 by Maxon Motor) run $10 \mathrm{KHz}$ PI current loops to regulate the current sent to the servo motors, $1 \mathrm{KHz}$ PI speed loop, and 1 $\mathrm{KHz}$ PID positioning loop.

\section{EVALUATION EXPERIMENTS}

Preliminary evaluation experiments were performed to assess the performance of the robotic system. A set of positioning trials, were conducted aiming at characterizing the system through quantitative measurements of its 
TABLE III. RESULTS FROM POSITIONING TRIALS

\begin{tabular}{lccccc}
\hline \hline Axis of Motion & $\mathbf{x}$ & $\mathbf{y}$ & $\mathbf{z}$ & $\boldsymbol{\vartheta}_{\boldsymbol{x}}$ & $\boldsymbol{\vartheta}_{\boldsymbol{y}}$ \\
\hline \# of Targets & 80 & 80 & 80 & 80 & 80 \\
Accuracy $^{\text {a }}$ & $1.18 \pm 1.14 \mathrm{~mm}$ & $1.12 \pm 0.90 \mathrm{~mm}$ & $0.09 \pm 0.01 \mathrm{~mm}$ & $0.32 \pm 0.11^{\circ}$ & $0.24 \pm 0.05^{\circ}$ \\
Repeatability & $0.16 \mathrm{~mm}$ & $0.10 \mathrm{~mm}$ & $0.04 \mathrm{~mm}$ & $0.10^{\circ}$ & $0.01^{\circ}$ \\
\hline \hline
\end{tabular}

positioning accuracy and repeatability. Each trial included repeated movement of the robot end-effector on a predefined grid of target poses within the robot workspace, i.e. $\pm 10 \mathrm{~mm} / \pm 15^{\circ}$ along/around each motion axis separately. 80 different target poses per axis were investigated for a total of 480 targets. An optical tracker (Polaris Spectra-NDI Inc.) acquired the actual pose of the robot end-effector at each target pose by tracking the optical markers placed on the robot according to a known geometry. The metric chosen for the positioning accuracy evaluation was the positioning rootmean-squared-error (RMSE); the metric chosen for the positioning repeatability evaluation was the standard deviation (SD) of the positioning errors on the repeated target positions. A 6-DOF load cell (FTSens by Istituto Italiano di Tecnologia, Italy) mounted on the robot endeffector was connected to a metal orthopedic pin (length 10 $\mathrm{cm})$. Two rubber bands were attached to the pin top end in order to simulate the force/torque required for fragment manipulation (see Table 1) ensuring that the reported positioning results have been achieved under realistic loading conditions. Force/torque data were also acquired during the trials. The experimental setup is shown in Fig.3a. The experimental results from the positioning trials are summarized in Table 3 and Table 4. The evaluation results show that the proposed system is accurate, presenting maximum residual positioning RMSE of only $1.18 \pm 1.14$ $\mathrm{mm}$ (translations along $\mathrm{x}$ ) and $1.85 \pm 1.54^{\circ}$ (rotations around z). Also, the system demonstrates sub-millimeter and subdegree repeatability with maximum values of $0.16 \mathrm{~mm}$ and $0.08^{\circ}$ when translating and rotating respectively along $\mathrm{x}$ and around $z$, as shown in Table 3 . The load measured during the positioning experiments resulted in average force of $20.74 \pm$ $0.2 \mathrm{~N}$ and average torque of $1.97 \pm 0.02 \mathrm{Nm}$ as summarized in Table 4.

TABLE IV. LOAD MEASUREd DURING POSITIONING TRIALS

\begin{tabular}{ll}
\hline \hline Parameter & Value \\
\hline Average Force Applied $\left(F_{x}, F_{y}, F_{z}\right)$ & $20.74 \pm 0.2 \mathrm{~N}$ \\
Average Torque Applied $\left(T_{x}, T_{y}, T_{z}\right)$ & $1.97 \pm 0.02 \mathrm{Nm}$ \\
\hline
\end{tabular}

\section{CONCLUSION}

This paper presented the design and implementation of a novel system for robot-assisted fracture manipulation. The aim of the paper was to improve the actual surgical procedure by providing the surgeon with a precise, minimally invasive, ergonomic and safe robotic system for fracture manipulation. This system allows for accurate fragment repositioning without open surgery, and obviates problems related to the current conventional surgical techniques, such as higher risk of infection, higher damage to soft tissues, and consequent prolonged period of postoperative rehabilitation. The system was evaluated through positioning trials to measure its accuracy and repeatability. The results reported in Table 3 and 4 showed that the new robotic system met the application requirements summarized in Table 1 achieving the level of accuracy and applied force/torque required for joint fracture manipulation. Also, these experiments demonstrate that the proposed system has a slightly higher level of accuracy and repeatability when compared with other systems based on parallel robot kinematics for long bone fracture reduction [7], [8]. The accuracy and the repeatability of the system could be further improved by realizing a vision-based position control of the robot [11]. This would reduce the current positioning deviations due to the manufacturing process, e.g. the manufacturing of laser-cut parts of the struts.

\section{ACKNOWLEDGMENT}

This is a summary of independent research funded by the National Institute for Health Research (NIHR)'s Invention for Innovation (i4i) Programme. The views expressed are those of the author(s) and not necessarily those of the NHS, the NIHR or the Department of Health.

\section{REFERENCES}

[1] Kulkarni GS, Textbook of orthopaedics and trauma. New Delhi: Jaypee Brothers, 2008.

[2] S. Rammelt, et al, "Percutaneous Treatment of Less Severe Intraarticular Calcaneal Fractures," Clin. Orthop., 468(4), 2010.

[3] Gaston P, et al, "Recovery of knee function following fracture of the tibial plateau," J Bone Joint Surg Br, 87(9) 2005.

[4] M. I. Klein, et al, "Mental Workload and Stress Perceived by Novice Operators in the Laparoscopic and Robotic Minimally Invasive Surgical Interfaces,” J. Endourol., 26(8), 2012.

[5] Berlinger NT, "Robotic surgery-squeezing into tight places," N Engl J Med, vol. 354, pp. 2009-2101, 2006.

[6] Westphal R, et al, "Robot-assisted Long Bone Fracture Reduction," The Int J of Robotics Res, 28, 2009.

[7] Wang J, et al, "Femoral fracture reduction with a parallel manipulator robot on a traction table," Int J Med Robotics Comput Assist Surg, 2013.

[8] Tang P, et al, "Novel 3D hexapod computer-assisted orthopaedic surgery system for closed diaphyseal fracture reduction," Int J Med Robot, 8(1), 2012.

[9] G. Dagnino, et al, "Force-Torque Measurement System for fracture surgery," CAOS 2015, Vancouver, Canada, 2015.

[10] I. Georgilas, et al, "Preliminary Analysis of Force-Torque Measurements for Robot-Assisted Fracture Surgery," EMBC 2015, Milan, Italy, 2015.

[11] G. Dagnino, et al, "Vision-Based Real-Time Position Control of a Semi-automated System for Robot-Assisted Joint Fracture Surgery," CARS 2015, Barcelona, Spain, 2015. 